\title{
Patrones de distribución y estructura de la vegetación en el gradiente de humedales costeros El Castaño, Chiapas, México
}

\author{
Matilde Rincón-Pérez ${ }^{1}$, Dulce Infante-Mata ${ }^{1,2 *}$, Patricia Moreno-Casasola ${ }^{2}$, María Elizabeth \\ Hernández Alarcón ${ }^{2}$, Everardo Barba Macías ${ }^{3}$ \& José Rubén García-Alfaro ${ }^{1}$ \\ 1. Manejo Sustentable de Cuencas y Zonas Costeras. El Colegio de la Frontera Sur. Carretera Antiguo Aeropuerto km \\ 2.5 s/n Tapachula, México; matilderinconperez@yahoo.com.mx, dulce.infante@gmail.com,jrgarcia@ecosur.mx \\ 2. Red de Ecología Funcional, Red de Manejo Biotecnológico de Recursos. Instituto de Ecología A.C. Xalapa, Veracruz, \\ México; patricia.moreno@inecol.mx, elizabeth.hernandez@inecol.mx \\ 3. Manejo Sustentable de Cuencas y Zonas Costeras. El Colegio de la Frontera Sur. Carretera a Reforma, km $15.5 \mathrm{~s} / \mathrm{n}$ \\ Ra. Guineo 2da Sección. C.P. 86280 Villahermosa, Tabasco, México; ebarba@ecosur.mx \\ * Correspondencia
}

Recibido 10-VI-2019. Corregido 06-XI-2019. Aceptado 27-II-2020.

\begin{abstract}
Distribution patterns and vegetation structure in the coastal wetland gradient in the Castaño, Chiapas, Mexico. Introduction: The characteristics of coastal wetlands are the result of hydrogeomorphological interactions between the continent and the ocean, which cause an environmental gradient, hat results in different vegetation types such as mangroves, freshwater marshes, swamp forests and palm swamps. Objective: To characterize the hydroperiod and physicochemical variables of water and soil and their effect on the distribution of vegetation in the Sistema de Humedales El Castaño. Methods: A total of 11 permanent sampling units (UM) were established by defined strata: five in the mangrove, two in swamp forest, two in freshwater marshes and two in the flooded pasture. From May 2016 to October 2017 the vegetation was characterized and the water levels and physicochemical parameters (superficial, interstitial and groundwater) were sampled monthly for: salinity, and $\mathrm{pH}$; and the soil for: bulk density, humidity percentage, and redox potential. Results: Mangroves are the closest to the sea, have the lowest diversity (H: 1.66) and species richness (14), they are dominated by Laguncularia racemosa and Rhizophora mangle, have the highest values of interstitial and groundwater salinity, (>10.8 ups), remain flooded for 4 to 12 months per year, and have a redox potential of $14.57 \mathrm{mV}$. Immediately, inland, there are remnants of the swamp forests (H: 2.18 and 18 species), dominated by Pachira aquatica, with 5 ups interstitial and groundwater salinity, flooded from 0 to 6 months per year, with a redox potential of $119.07 \mathrm{mV}$. These forests are followed inland by freshwater marshes (H: 1.92 and 16 species), dominated by Typha domingensis with 6.1 ups interstitial and groundwater salinity, flooded for 5 to 8 months per year and a redox potential of $125.9 \mathrm{mV}$. Finally, furthest inland is the flooded pasture, a modified herbaceous wetland for cattle grazing (H: 3.44 and 50 species) dominated by Paspalum conjugatum, where interstitial and groundwater salinity is less than 0.5 ups, it stays flooded for 5 to 9 months and the redox potential is 151.23 $\mathrm{mV}$. Conclusions: In each type of vegetation, the structure, composition, and diversity are different, with a high turnover of species that indicates a gradient defined by salinity. The vegetation in the SHC follows the patterns of typical organization of the tropical coastal wetlands, mangroves, swamp forests and herbaceous wetlands, in this case the freshwater marshes and flooded pastures. The factor that define the distribution of the vegetation is the salinity and the gradient that is observed are a function of the hydrological dynamics that depends on the mixing of marine and freshwater.
\end{abstract}

Key words: environmental gradients, hydrophytes, tropical peat, modified wetland, hydrology, diversity, connectivity.

Rincón-Pérez, M., Infante-Mata, D., Moreno-Casasola, P., Hernández Alarcón, M. E., Barbas Macías, E., \& García-Alfaro J. R. Patrones de distribución y estructura de la vegetación en el gradiente de humedales costeros El Castaño, Chiapas, México. Revista de Biología Tropical, 68(1), 242-259. 
Existen diferentes humedales definidos por la interacción del agua, el suelo hídrico y la vegetación hidrófita, que junto con la geomorfología y el clima resultan en distinta composición y estructura de la vegetación (Wheeler, Money, Shaw, Perrow, \& Davy, 2002; Mitsch \& Gosselink, 2007). El interés por entender su funcionamiento ha aumentado debido al reconocimiento de los servicios ecosistémicos que proveen y su papel clave en la mitigación y adaptación al cambio global (Costanza et al., 1997; MEA, 2005; de Groot et al., 2012). Sin embargo, el cambio climático y el cambio de uso de suelo están modificando sus características bióticas y abióticas, alterando procesos funcionales como el almacenamiento de agua, la productividad y el almacenamiento de carbono (Bergh et al., 2001).

La vegetación, es un componente de los humedales, y los patrones de la distribución de las especies que la componen están relacionadas con los factores ambientales como el tipo de suelo, la disponibilidad de nutrientes, el hidroperiodo, la topografía que han sido estudiados por diversos autores (Zeilhofer \& Schessl, 1999; Elmore, Mustard, \& Manning, 2003; Crain, Silliman, Bertness, \& Bertness 2004; King, Richardson, Urban, \& Romanowicz, 2004; Givnish et al., 2008; Crase, Liedloff, Vesk, Burgman, \& Wintle, 2013). En los humedales costeros, las características hidrogeomorfológicas definen las interacciones entre el ambiente terrestre y oceánico (de la Lanza Espino, Pérez, \& Pérez, 2013) que condicionan las variaciones de los factores ambientales como la salinidad y la inundación, considerados los principales factores que determinan la composición, estructura y diversidad de la vegetación (Brinson, Bradshaw, \& Jones, 1985; Carreño, Esteve, Martínez, Palazón, \& Pardo, 2008; Anderson \& Lockaby, 2011; Courtwright, \& Findlay, 2011).

El hidroperiodo del humedal es el patrón estacional del nivel del agua, resultado del balance entre las entradas y salidas de agua, la geomorfología y el suelo, donde los procesos biogeoquímicos, como la reducción del hierro, magnesio y bióxido de carbono, se rigen por las reacciones de óxido-reducción (redox) influenciados por el tiempo de inundación (Mitsch \& Gosselink, 2007; Vepraskas \& Faulkner, 2001). La salinidad en los humedales costeros está determinada por el hidroperiodo, las fluctuaciones de la marea, la salinidad del agua del mar, los cambios en el nivel del mar, los aportes de agua dulce de los ríos, la precipitación y el agua subterránea (Zedler \& Callaway, 2001; Barendregt \& Swarth, 2013).

La relación entre los humedales costeros se ha evaluado con base a la conectividad hidrológica (Zedler \& Callaway, 2001; Davis, Childers, Lorenz, Wanless, \& Hopkins, 2005). Poco se sabe sobre la conectividad estructural (vegetación), la mayoría de los estudios están referidos a un tipo de humedal (e.g. manglar, selva inundable) (Agraz-Hernández, GarcíaZaragoza, Iriarte-Vivar, Flores-Verdugo, \& Moreno-Casasola, 2011; Infante-Mata, Moreno-Casasola, Madero-Vega, Castillo-Campos, \& Warner, 2011; Moreno-Casasola et al., 2010) y reportan la diversidad alfa, con excepción de algunos trabajos que estiman la diversidad beta para conocer el recambio de las especies y la conectividad que puede existir entre estos humedales heterogéneos donde existe un gradiente ambiental que se refleja en un cambio en la estructura y composición de la vegetación (Rickert, Fichtner, van Klink, \& Bakker, 2012; Socolar, Gilroy, Kunin, \& Edwards, 2016; Aponte, 2017). En general la diversidad vegetal de los humedales costeros es baja y dominada por algunas especies, debido a que son pocas las plantas con adaptaciones morfológicas y fisiológicas, para crecer en zonas inundadas y bajo influencia salina (Hackney \& Avery, 2015; Lee, Jones, Diele, Castellanos-Galindo, \& Nordhaus, 2017).

En México, Chiapas ha perdido el $57 \%$ de humedales, principalmente los costeros de agua dulce (Landgrave \& Moreno-Casasola, 2012; Valderrama-Landeros et al., 2017). Las actividades antrópicas a lo largo de las cuencas costeras afectan a la planicie, causando el deterioro del hábitat, la modificación de los procesos funcionales (e.g. productividad, almacenamiento de carbono) y disminuyendo la conectividad 
entre los humedales costeros de Chiapas (Contreras-Espinosa \& Warner, 2004; Calva, Pérez, \& Márquez, 2006; de la Fuente, Lisle, \& McKean, 2007; Alonso-Eguialis et al., 2013).

La planicie costera de Chiapas es angosta, con un ancho entre 15 y $35 \mathrm{~km}$, con un relieve bajo que se eleva abruptamente a casi 2000 msnm. La precipitación anual es de 1500 a $2000 \mathrm{~mm}$ a lo largo de la planicie y de 2100 a más de $4000 \mathrm{~mm}$ en la parte alta. Las características hidrogeomorfológicas de los ríos (pendiente, topografía, caudal, sinuosidad, entre otros), determinan la distribución, la dimensión y la morfología de los humedales costeros (Lisle, De la Fuente, Suarez, \& Hernández, 2009; de la Fuente et al., 2007; Pérez, 2013). Los humedales que se observan del mar hacia tierra adentro son: (1) el manglar, que cubre una gran extensión y se conecta horizontalmente a través de los canales y los esteros con los principales cuerpos lagunares (ValderramaLanderos et al., 2017); (2) la selva inundable, que ocupa una extensión mucho menor que el manglar, y forma parches remanentes de pocas hectáreas en el Sistema de Humedales el Castaño (SHC) y la desembocadura del río Huixtla (Breedlove, 1981; Rincón, 2014); (3) los humedales herbáceos, tulares y popales, que ocupan también grandes extensiones y mantienen conectividad horizontal a través de canales (Rzedowski, 1978; SEMARNAP, 1999); y (4) humedales modificados como los pastizales inundables, que son áreas ocupadas para uso ganadero (Liscovsky, Parra, Balente, Huerta, \& Ramos, 2009).

En este trabajo se aborda el estudio del SHC para entender el funcionamiento (e.i. la forma en que interactúan los factores bióticos y abióticos, su relación con la distribución de la vegetación y el mantenimiento de los procesos ecológicos) de este tipo de sistema de humedales costeros. El objetivo fue caracterizar las variables del hidroperiodo, las características fisicoquímicas del agua y el suelo para determinar su relación con la distribución de la vegetación de los humedales. Se plantean las siguientes hipótesis: i) Se espera encontrar un gradiente ambiental definido por la inundación y salinidad de mayor a menor influencia marina al aumentar los aportes de agua dulce del continente, que se reflejará en un cambio en la estructura y la composición de la vegetación; ii) la diversidad alfa variará de menor a mayor al disminuir la inundación y la salinidad; la diversidad beta (recambio de especies) será mayor entre las comunidades vegetales con mayor diferencia de inundación y salinidad.

\section{MATERIALES Y MÉTODOS}

Área de estudio: El SHC forma parte de la región hidrológica prioritaria para la conservación 32, de acuerdo con la Comisión Nacional para el Conocimiento y Uso de la Biodiversidad (CONABIO) (SEMARNAP, 1999; Tovilla et al., 2007), se localiza en la planicie costera del Pacífico Sur, en donde desembocan los ríos San Nicolás, Sesecapa, Madre Vieja y Bonanza-Camargo y forma parte del humedal El Castaño-Barra Zacapulco-Laguna Campón y Chantuto, al NW de la zona núcleo de la Reserva de La Biósfera La Encrucijada (REBIEN) $\left(15^{\circ} 16^{\prime} 51.93\right.$ " N \& 92 $57^{\circ} 55.02$ " W) entre las coordenadas $15^{\circ} 17^{\prime} 7.72^{\prime \prime} \mathrm{N} \& 92^{\circ} 56^{\prime} 57.76^{\prime \prime} \mathrm{W}$ y $15^{\circ} 15^{\prime} 40.56$ ” N \& 92 $57^{\circ} 31.47^{\prime \prime}$ W (Fig. 1). El clima es cálido-húmedo, con abundantes lluvias en verano (Am) (García, 2004), la precipitación anual es de $1567 \mathrm{~mm}$ y temperatura media anual de $28.2{ }^{\circ} \mathrm{C}$ con un mínimo de 19.2 ${ }^{\circ} \mathrm{C}$ y un promedio anual máximo de $36.5{ }^{\circ} \mathrm{C}$ (CONAGUA-SMN, 2018). La vegetación es característica de la REBIEN y está formada por selva baja caducifolia, vegetación de dunas costeras, palmares, popales, tulares, manglares, selvas inundables, vegetación flotante y subacuáticas (Rzedowski, 1978; Breedlove, 1981), así como potreros y cultivos diversos.

Método: Con base al conocimiento previo de la zona y con apoyo del visualizador Google Earth se propusieron los sitios de muestreo. Se realizaron dos recorridos de verificación para ubicar los tres tipos de humedales naturales (manglar, selva inundable y tular), y un humedal modificado por el uso ganadero (pastizal inundable). Se establecieron en total 11 unidades 


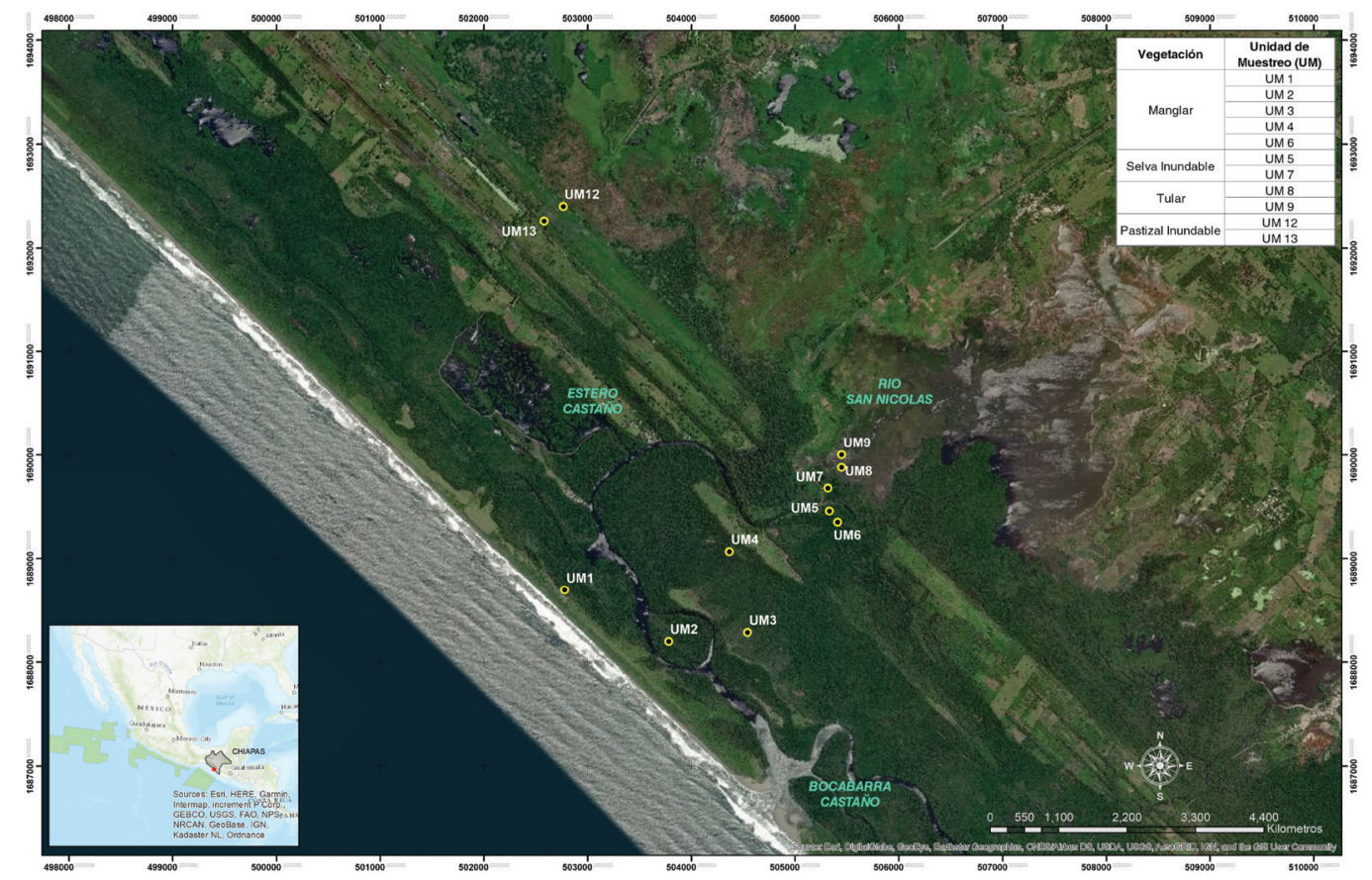

Fig. 1. Área de estudio, Sistema de Humedales El Castaño, Chiapas, México.

Fig. 1. Study site, El Castaño Wetlands System, Chiapas, Mexico.

de muestreo (UM) permanentes por humedal: cinco en el manglar, dos en la selva inundable, dos en el tular y dos en el pastizal inundable. En el manglar se establecieron cinco UM porque la superficie que cubre es mayor (Carranza \& Molina, 2003) y presenta una mayor heterogeneidad (Romero-Berny, Tovilla-Hernández, Torrescano-Valle, \& Schmook, 2019). El muestreo es intencional y dirigido como lo sugiere Fourqurean et al. (2014). En todas las UM se caracterizó la vegetación (al inicio del trabajo) y en cada parcela se muestreó mensualmente de mayo 2016 a octubre 2017, el tiempo (meses) y el nivel de inundación; y de noviembre 2016 a octubre 2017, los parámetros fisicoquímicos del agua (superficial, intersticial y subterránea) y del suelo.

Hidroperiodo (nivel y tiempo de inundación): Se midió el nivel del agua en 52 piezómetros, construidos e instalados considerando el método de Yetter (2004) y Peralta-Pélaez, Infante-Mata y Moreno-Casasola (2009). Para conocer el hidroperiodo se graficaron por piezómetro los valores mensuales con respecto al nivel del suelo. El nivel de inundación de cada piezómetro es independiente de otro, ya que no fueron calibrados con respecto a un punto de referencia común, como el nivel del mar.

Parámetros fisicoquímicos: Se midió la salinidad y el $\mathrm{pH}$ del agua con un multiparámetro YSI Mod. 85 y un sensor de $\mathrm{pH}$ eco Testr modelo $\mathrm{pH} 2$ (Oakton ${ }^{\mathrm{TM}}$ ). Los datos de agua superficial se tomaron directamente del espejo de agua, el agua intersticial entre $15-20 \mathrm{~cm}$ del suelo y el agua subterránea a $150 \mathrm{~cm}$ del suelo, siguiendo los métodos sugeridos por InfanteMata, Peralta-Pélaez y Arrocha (2009).

Se recolectaron muestras de los primeros 8 cm de suelo con un cilindro de cobre (190.07 $\mathrm{cm}^{3}$ ). Siguiendo el método de Wilke (2005) se obtuvo la densidad aparente y el porcentaje de humedad. El potencial redox (Eh) se midió utilizando tres electrodos de platino construidos con el método de López-Rosas 
y Tolome (2009), que incluye la calibraron y ajuste a los cálculos.

Estructura y composición de la vegetación: Para la caracterización de la vegetación se utilizaron los métodos sugeridos por Moreno-Casasola y Warner (2009) y Kauffman, Donato y Adame (2013). En el manglar y la selva inundable se establecieron cinco parcelas circulares de $12.6 \mathrm{~m}$ de radio sumando $2500 \mathrm{~m}^{2}$ por UM. En el tular y el pastizal inundable fueron cinco parcelas rectangulares de $25 \mathrm{x}$ $20 \mathrm{~m}$ cada una, sumando $2500 \mathrm{~m}^{2}$ por UM; esta parcela se dividió en subparcelas de 5 x $5 \mathrm{~m}$ y se muestrearon 10 subparcelas. En cada UM se identificaron las especies en campo y en caso de no definirla se identificó posteriormente con apoyo de guías y ejemplares de herbario de ECOSUR-Tapachula.

En la vegetación arbórea se registró el diámetro a la altura del pecho (DAP) de los individuos mayores de $2.5 \mathrm{~cm}$. Para los árboles multicaules cada tallo fue considerado como un individuo. Para cada especie de la vegetación arbórea y herbácea, se estimó la cobertura usando la escala de cobertura-abundancia de Westhoff y van der Maarel (1978).

Análisis de la diversidad, estructura y dominancia: Se obtuvo la curva de acumulación de especies y dos pruebas no paramétricas de Chao 2 y Jack-knife 1 para tener dos referencias al evaluar la representatividad del esfuerzo de muestreo de la riqueza. Para la diversidad alfa se obtuvo el índice de Shannon-Wiener (H') y el índice de Simpson (D-1), puesto que son los más utilizados para comparar la diversidad entre estudios, además del índice de equidad de Pielou (J'). La diversidad beta, se evaluó con el índice de Sorensen. Se elaboró un histograma de frecuencia con las clases diamétricas de la vegetación arbórea de manglar y selva inundable. Se calculó el Índice de Valor de Importancia (IVI) considerando la suma de la frecuencia relativa, densidad relativa y dominancia relativa para la vegetación arbórea y en el caso de la vegetación herbácea la frecuencia relativa y la cobertura relativa.
Análisis de datos ambientales: Para verificar la normalidad de los datos se utilizó la prueba de Kolmogorov-Smirnov. Para las comparaciones de las variables entre los cuatro humedales y por temporada se utilizó la prueba de Kruskal-Wallis (H), ya que los datos no presentaron una distribución normal. Para las variables que presentaron diferencias significativas $(\mathrm{P}<0.05)$ entre los humedales se realizaron pruebas de comparaciones múltiples de Tukey no paramétrica. Se realizaron las mismas comparaciones para la temporada de secas (noviembre 2016 a abril 2017) y lluvias (mayo a octubre de 2017). Para identificar diferencias entre el hidroperiodo y la precipitación se utilizó la prueba de correlación de Spearman.

Para establecer la forma en que se ordena espacialmente la vegetación de los sitios de muestreo e identificar las variables ambientales, que explicarán el gradiente ambiental, se hizo un análisis de escalamiento multidimensional no paramétrico (NMDS), que se considera el más adecuado ya que no asume linealidad, y se uso la distancia de BrayCurtis, que da a las especies comunes y raras pesos relativamente similares. La matriz que se utilizó para esta prueba fue la de coberturaabundancia por especie y parcelas. Para la mayoría de los análisis se utilizó el programa PAST v 3.2 (Hammer, Harper, \& Ryan, 2001), las comparaciones múltiples y los análisis de Spearman se realizaron con el programa $\mathrm{R}(\mathrm{R}$ Core Team, 2019).

\section{RESULTADOS}

Hidroperiodo (nivel y tiempo de inundación): Las fluctuaciones de nivel de agua observadas en los humedales, siguen el patrón de la precipitación de la región (Fig. 2A, Fig. 2B). Los niveles aumentan en junio, al inicio de las lluvias, y bajan al disminuir la precipitación en noviembre. Cada humedal presenta una variación diferente en nivel y tiempo de inundación. En el nivel de inundación hay diferencias significativas $(\mathrm{H}=62.16, \mathrm{P}<0.05$, Tukey, $\mathrm{P}<0.05$ ), el pastizal inundable presenta los niveles más bajos en secas, pero en 
lluvias alcanza un nivel similar al del manglar y tular, siendo la selva inundable la de menor inundación $(\mathrm{H}=36.15, \mathrm{P}<0.05)$. El manglar y el pastizal permanecieron inundados el mismo tiempo. En el manglar en las UM1, UM3 y
UM4 se observaron parcelas con inundación permanente. En relación al tiempo de inundación el manglar, el tular y el pastizal inundable permanecen mayor tiempo inundados con respecto a la selva inundable $(\mathrm{kw}=24.39$,

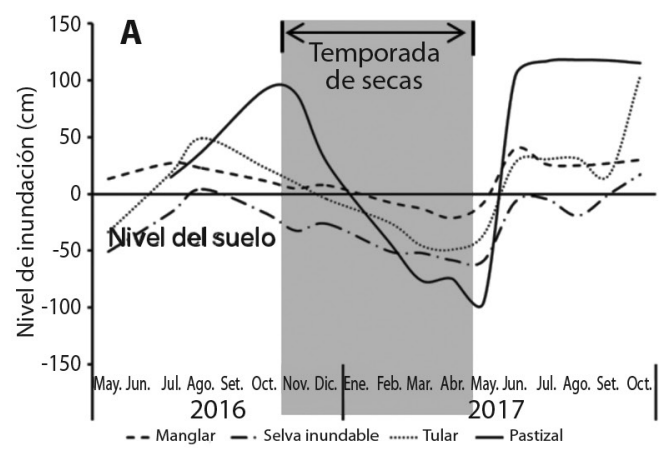

Hidroperiodo en las comunidades vegetales
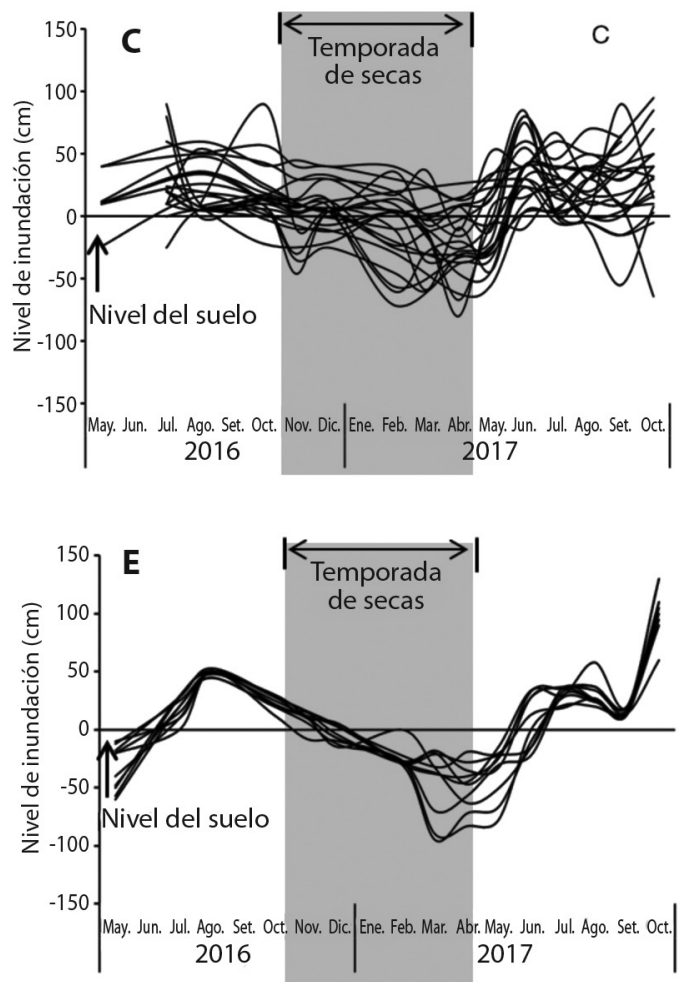

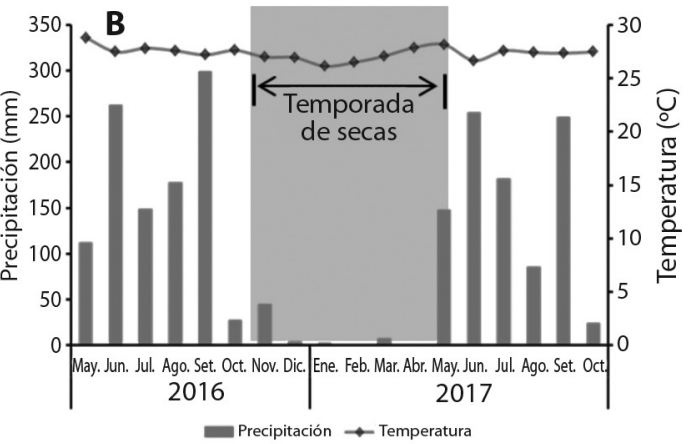

Estación climatológica EMA 15CA16EE
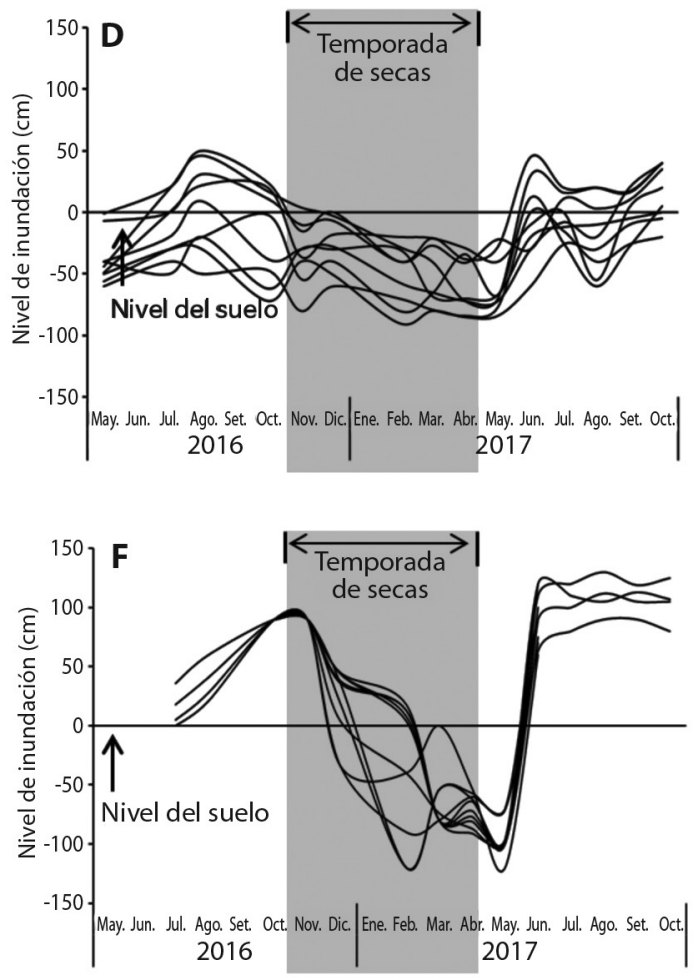

Fig. 2. Hidroperiodos. A. Hidroperiodo general de humedales; B. Precipitación y temperatura C. Manglar; D. Selva inundable, E. Tular y F. Pastizal inundable, en el Sistema de Humedales el Castaño. Cada línea de las gráficas corresponde a un piezómetro.

Fig. 2. Hydroperiods. A. General wetland hydroperiod; B. Precipitation and temperature C. Mangrove; D. Freshwater swamp, E. Freshwater marsh and F. Flooded grassland, in El Castaño Wetland System. Each line of the graphs corresponds to a piezometer. 
$\mathrm{P}<0.05$, Tukey, $\mathrm{P}<0.05$ ) (Apéndice Digital 1, Tabla A, Tabla B, Tabla C).

En el tular y el pastizal inundable, el aumento del nivel del agua se relaciona con la precipitación, (Spearman, $\mathrm{P}>0.05$ ). El manglar y la selva inundable presentaron un patrón diferente con la precipitación (Spearman, $\mathrm{P}<0.05$ ). En el manglar de mayo a octubre (lluvias), la inundación es generalizada y en noviembre (secas), disminuye, llegando al nivel más bajos en el mes de abril (Fig. 2C). En la selva inundable, aún cuando el nivel del agua subterránea aumenta en lluvias, no hay agua superficial en la mitad de las parcelas, y a partir de noviembre el agua llega a $-90 \mathrm{~cm}$ por debajo del suelo (Fig. 2D). En el tular las fluctuaciones en la temporada de lluvia son similares en todos los sitios; el agua superficial se mantuvo hasta diciembre, y en los siguientes meses baja a $-95 \mathrm{~cm}$ del suelo (Fig. 2E). En el pastizal inundable, el agua superficial sube hasta $120 \mathrm{~cm}$ y se mantiene en algunas parcelas hasta febrero; en los siguientes meses el nivel del agua desciende a $-120 \mathrm{~cm}$ por debajo del suelo (Fig. 2F).

Parámetros fisicoquímicos del agua: Los humedales del SHC difieren en los parámetros fisicoquímicos del agua. La salinidad del agua superficial del manglar, la selva inundable y el tular son mayores a la del pastizal inundable $(\mathrm{H}$ $=52.42, \mathrm{P}<0.05$, Tukey, $\mathrm{P}<0.05)$. En el agua intersticial y subterránea, la salinidad, del manglar es mayor a las otras comunidades. Entre la selva inundable y el tular la salinidad es igual y la del pastizal es menor que todas, durante todo el año. La salinidad del agua intersticial del tular durante la temporada de lluvia es diferente de la selva inundable, y el pastizal inundable. Con respecto al $\mathrm{pH}$, en el agua superficial del manglar y en el pastizal inundable, es diferente a la de la selva inundable y la del tular. No hay diferencias del $\mathrm{pH}$ del agual intersticial y subterránea, entre la del manglar y la de la selva inundable. El pH del tular es diferente al manglar en los tres tipos de agua. Durante la temporada de lluvias el pH del agua subterránea solo es diferente en el pastizal inundable (Apéndice Digital 1, Tabla A, Tabla B, Tabla C).

Parámetros fisicoquímicos del suelo: De las variables del suelo, el porcentaje de humedad de cada humedal fue diferente $(\mathrm{H}=54.20$, $\mathrm{P}<0.05$, Tukey, $\mathrm{P}<0.05$ ); los valores más altos se registraron en el manglar $(90.76 \%)$ y los más bajos en el pastizal inundable (7.7\%). En la temporada de secas la humedad del suelo de la selva inundable y el pastizal inundable, fueron similares $(\mathrm{H}=26.83, \mathrm{P}<0.05$, Tukey, $\mathrm{P}<0.05)$. Con respecto a la densidad aparente del suelo solo el pastizal inundable y la selva fueron diferentes $(\mathrm{H}=16.24, \mathrm{P}<0.05$, Tukey, $\mathrm{P}<0.05)$. El potencial redox, considerando el promedio anual, para el manglar fue diferente a los otros humedales $(\mathrm{H}=40.84, \mathrm{P}<0.05$, Tukey, $\mathrm{P}<0.05)$. En secas el potencial redox del manglar es igual al de la selva inundable pero difieren con respecto a los humedales herbáceos $(\mathrm{H}=25.22, \mathrm{P}<0.05$, Tukey, $\mathrm{P}<0.05)$. En temporada de lluvias el potencial redox de la selva inundable es mayor con respecto al de los otros humedales $(\mathrm{H}=21.15, \mathrm{P}<0.05$, Tukey, $\mathrm{P}<0.05$ ) (Apéndice Digital 1, Tabla A, Tabla B, Tabla C).

Composición y diversidad de la vegetación: Se registraron 78 especies pertenecientes a 33 familias. El pastizal inundable tiene la mayor riqueza (50), seguido por la selva inundable (18), el tular (16) y el manglar (14). De las 78 especies registradas, 53 son hierbas, 12 son árboles, seis pueden ser encontradas en forma de árboles o arbustos, hay una trepadora, dos pueden crecer como arbusto y trepador, tres como hierba y trepador y una epífita. Según la CONABIO (2016), 45 especies tienen hábitats terrestres, 16 dulceacuícolas, 11 en dulceacuícolas y terrestres, tres en dulceacuícolas y salobres, y tres en dulceacuícolas, salobres y terrestres (Apéndice Digital 2).

A nivel nacional, considerando la base de datos de la CONABIO (2016), se registraron dos especies endémicas (Hampea trilobata y Sabal mexicana), y seis especies exóticas Cucumis anguria, Eichhornia crassipes, 
Elaeis guineensis, Brachiaria decumbes, Cynodon dactylon y Panicum maximum, siendo las últimas tres pastos.

Las especies del manglar ( $R$. mangle, $L$. racemos, $A$. germinans y $C$. erectus), tienen categoría de amenazadas (A) por la NOM059 en México. También se identificaron 21 especies evaluadas por la Unión Internacional para la Conservación de la Naturaleza (UICN) en categoría de bajo riesgo, preocupación menor (LR(lc)) (Apéndice Digital 2) (UICN, 2017).

La curva de acumulación de especies no llegó a la asíntota (Apéndice Digital 3), pero se obtuvieron los porcentajes de representatividad de las especies esperadas con los estimadores de Chao2 y Jack-knife1 (Tabla 1). Con el primer estimador la representatividad fue de $93 \%$ para manglar, $95 \%$ la selva inundable, $84 \%$ el tular y $75 \%$ el pastizal inundable. Con el segundo estimador, los porcentajes observados fueron, $78 \%$ en el manglar, $82 \%$ la selva inundable, $80 \%$ el tular y $75 \%$ el pastizal. Con los resultados de ambos estimadores se confirmó la observación mínimo del $75 \%$ de las especies esperadas.

Para la diversidad alfa el mayor valor se obtuvo en el pastizal inundable (H: 3.44, D-1: $\left.0.96, J^{\prime} 0.88\right)$, seguido por la selva inundable (H: 2.18, D-1:0.85, J'0.75), el tular (H: 1.92, D-1: 0.76, J'0.69) y el menor el manglar, $(\mathrm{H}$ : 1.66, D-1:0.74, J'0.63). El índice de Sorensen, indica un menor recambio de especies entre los humedales arbóreos (el manglar y la selva inundable), al presentar una mayor similaridad de 0.56 compartiendo nueve especies. Entre los humedales herbáceos (el tular y el pastizal inundable) la similaridad fue de 0.21 el recambio es mayor, se comparten siete especies. Entre los humedales arbóreos y herbáceos el cambio de especies es mayor, entre el manglar y el pastizal inundable la similaridad es de 0.06 , solo comparten dos especies. Hay tres especies que se comparten en tres humedales: Zygia conzattii y Cissus sicyoides que se encuentra en el manglar, selva inundable y pastizal inundable, y Acrostichum aureum, que se encuentran en el manglar, selva inundable y tular. En general cada tipo de humedal tiene especies vegetales características que los definen, y no hay especies que se encuentren en los cuatro humedales (Tabla 2, Apéndide Digital 1).

TABLA 2

Índice de diversidad beta de Sorensen

TABLE 2

Sorensen beta diversity index

\begin{tabular}{lccc} 
& $\begin{array}{c}\text { Selva } \\
\text { Inundable }\end{array}$ & Tular & $\begin{array}{c}\text { Pastizal } \\
\text { Inundable }\end{array}$ \\
Manglar & 0.56 & 0.07 & 0.06 \\
Selva Inundable & & 0.12 & 0.09 \\
Tular & & & 0.21 \\
\hline
\end{tabular}

Estructura de la vegetación: En el manglar, se registraron 1945 ind ha ${ }^{-1}$ y $20.50 \mathrm{~m}^{2}$ $\mathrm{ha}^{-1}$ de área basal. Las especies con el mayor IVI fueron L. racemosa y $R$. mangle con 117.91 $\%$ y $116.14 \%$, respectivamente. En la selva inundable se estimó una densidad de 3256 ind ha-1 y un área basal de $23.64 \mathrm{~m}^{2} \mathrm{ha}^{-1}$. La especie con el mayor IVI es $P$. aquatica (Apéndice Digital 4, Tabla A). La distribución de las clases diamétricas de individuos del manglar y selva inundable siguió una tendencia similar,

TABLA 1

Área muestreada por sitio, especies observadas y estimadas según los índices Chao2 y Jack-knife1

TABLE1

Area sampled by site, species observed and estimated according to the Chao2 and Jack-knife1 indexes

\begin{tabular}{lcccc}
\multicolumn{1}{r}{ Vegetación } & Área $\left(\mathrm{m}^{2}\right)$ & Especies observadas & Estimada Chao2 & Estimada Jack-knife1 \\
Manglar & 12000 & 14 & 15 & 18 \\
Selva Inundable & 5000 & 18 & 19 & 22 \\
Tular & 5000 & 16 & 19 & 20 \\
Pastizal Inundable & 5000 & 50 & 67 & 67 \\
\hline
\end{tabular}




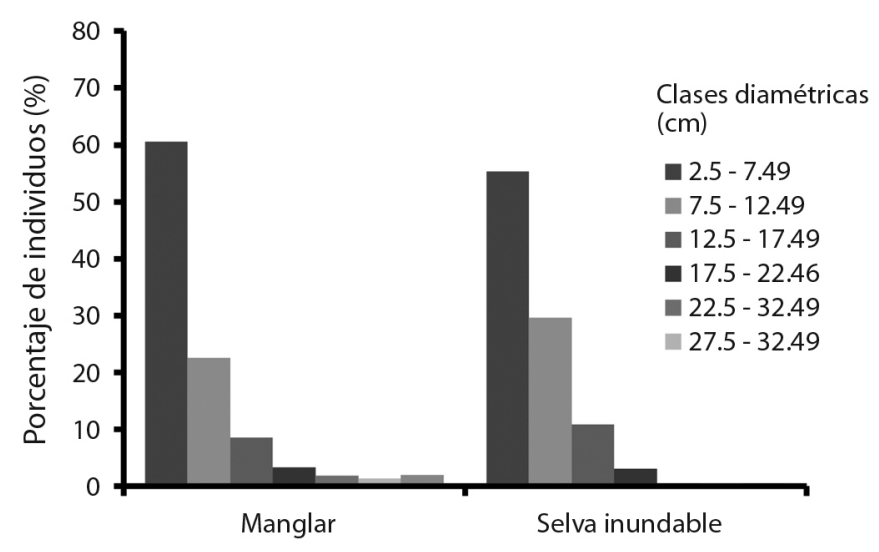

Fig. 3. Porcentaje de individuos en cada clase diamétrica del manglar y de la selva inundable del Sistema de Humedales el Castaño.

Fig. 3. Percentage of individuals in each diametric class of the mangrove and freshwater swamp of the El Castaño Wetlands System.

disminuyendo hacia las clases de mayor diámetro. En ambos casos son más frecuentes los individuos con diámetros entre $2.5 \mathrm{y}$ $7.49 \mathrm{~cm}$ (Fig. 3).

En los humedales herbáceos, T. domingensis domina en el tular con $85.2 \%$ de IVI; los valores del IVI están por debajo del $20 \%$ en el resto de las especies. En el pastizal inundable $P$. conjugatum es la especie dominante con 21.57 $\%$ de IVI seguida por Solanum hirtum con $13.62 \%$ y el resto; de las especies tiene valores de IVI menores al $10 \%$ (Apéndice Digital 4, Tabla B y Tabla C).

Distribución de la vegetación y su relación con las variables ambientales. Los resultados del análisis de escalamiento multidimensional con un stress 0.3136 , muestran que la composición de las especies es diferente entre las comunidades vegetales por la distribución de las UM a lo largo del eje $1\left(\mathrm{R}^{2}\right.$ $=0.5284$ ), ordenando, al manglar, la selva inundable, el tular y al pastizal inundable a lo largo de un gradiente definido por la salinidad del agua subterránea e intersticial y potencial redox (Fig. 4).

El manglar, dominado por R. mangle, $L$. racemosa y $A$. germinans, ocupa el extremo izquierdo de la ordenación con los mayores valores de salinidad. La selva inundable, dominada por $P$. aquatica, con elementos de $R$. mangle y $Z$. conzattii, y el tular, compuesto por $T$. domingensis, como especie principal, y con menor presencia, Sesuvium maritimun y A. aureum, en el centro de la ordenación; y el pastizal inundable a la derecha con la menor salinidad. Las tres principales especies que lo componen son $P$. conjugatum, Solanum hirtum y $B$. decumbens. Por lo tanto, el eje 1 muestra un gradiente de salinidad y sobre el eje $2\left(\mathrm{R}^{2}=\right.$ 0.2606 ) se establece un gradiente definido por el $\mathrm{pH}$ intersticial, y el porcentaje de humedad del suelo. El tular tiene los valores más bajos de $\mathrm{pH}$ y el más alto porcentaje de humedad del suelo, y el tiempo de mayor inundación se registra en el manglar y en el pastizal inundable (Fig. 4).

Distribución de las comunidades vegetales en el SHC a lo largo del gradiente ambiental definido principalmente por la salinidad (Fig. 5).

\section{DISCUSIÓN}

El gradiente ambiental del SHC está definido por la salinidad, de mayor a menor, principalmente del agua subterránea, a través de los humedales de la costa hacia tierra adentro 


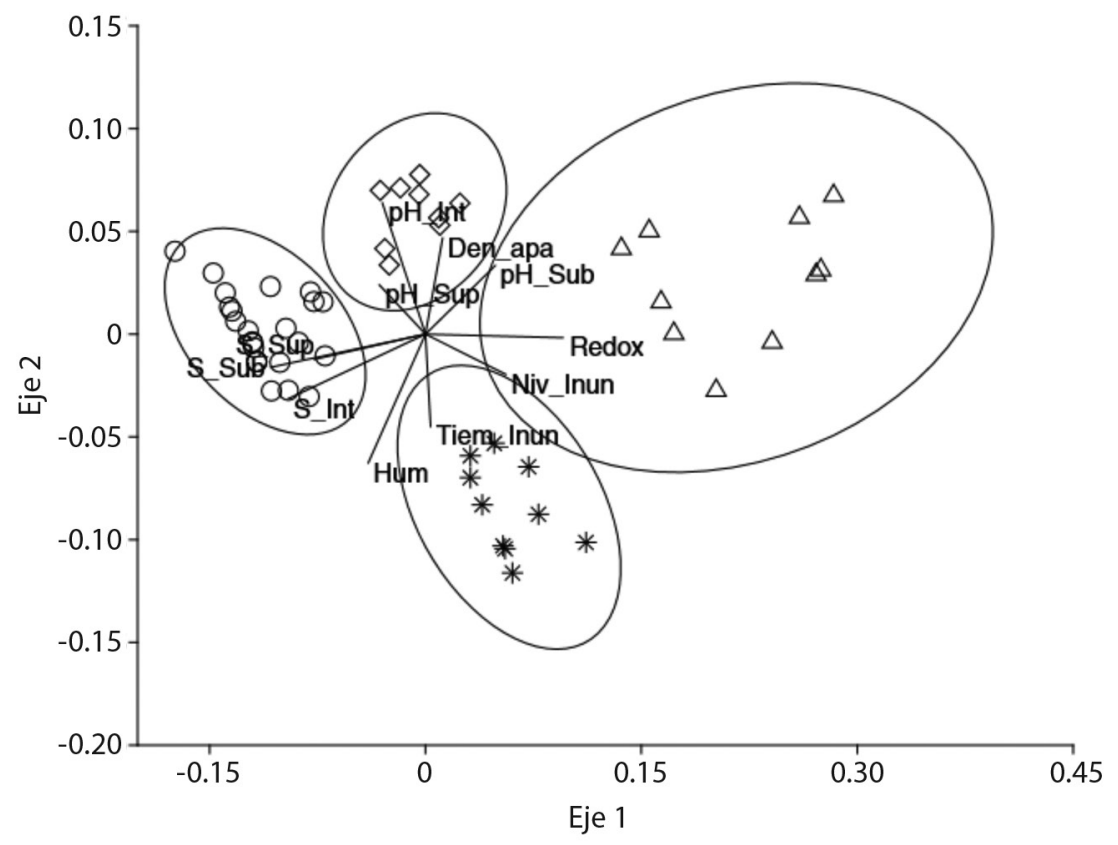

O Manglar $\diamond$ Selva inundable $\square$ Tular $\Delta$ Pastizal inundable.

Fig. 4. Análisis de escalamiento multidimensional no paramétrico de las especies de plantas usando distancia de Bray-Curtis que muestra la relación entre las comunidades vegetales y las variables ambientales. Salinidad superficial (S_Sup), salinidad intersticial (S_Int), salinidad subterránea (S_Sub), pH superficial ( $\mathrm{pH}$ _Sup), pH intersticial ( $\mathrm{pH}$ Int), $\mathrm{pH}$ subterránea (pH_Sub), porcentaje de humedad (Hum), densidad aparente (Den_apa), nivel inundación (Niv_Inun), tiempo de inundación (Tiem_Inun), Potencial Redox (Redox).

Fig. 4. Non-metric multidimensional scaling analysis of plant species using Bray-Curtis distance that shows the relationship between plant communities and environmental variables. Surperficial salinity (S_Sup), interstitial salinity (S_Int), groundwater salinity (S_Sub), surperficial $\mathrm{pH}$ ( $\mathrm{pH} \_$Sup), interstitial $\mathrm{pH}$ ( $\mathrm{pH}$ _Int), groundwater $\mathrm{pH}$ ( $\mathrm{pH}$ _Sub), humidity percentage (Hum), bulk density (Den_apa), level flood (Niv_Inun), flood time (Tiem_Inun), Redox Potential (Redox).

durante todo el año. El nivel de inundación, no presentó el gradiente como se esperaba, debido a que el manglar y el pastizal inundable, los humedales más alejados entre sí, mantienen el mismo tiempo de inundación y el nivel del agua durante la temporada de lluvias. Esto se explica por el origen de las aportaciones de agua al sistema. En el pastizal, el humedal más alejado del mar, la influencia marina es poca, el agua llega por la precipitación, el desbordamiento de los ríos y el agua subterránea, por lo que permanece inundado durante la temporada de lluvias y parte de la temporada de secas por los aportes del agua subterránea que continúan fluyendo. Por lo que la primera hipótesis se cumple parcialmente, puesto que sólo la salinidad presenta un gradiente de mayor a menor al aumentar los aportes de agua dulce. Con respecto al potencial redox, el $\mathrm{pH}$, la densidad aparente y el porcentaje de humedad en el suelo, el gradiente que se observa está sujeto a cambios según la temporada del año.

La diferencia en la salinidad del agua entre los humedales es uno de los factores que limita la distribución, establecimiento y crecimiento de las especies. El agua intersticial y subterránea es la que tienen disponible las plantas. En el manglar la zonación de las especies depende de la salinidad intersticial, y cada especie tiene diferente rango de tolerancia y adaptaciones que les permite vivir hasta en 80 ups (LópezPortillo et al., 2011). Los valores en el SHC para el manglar están dentro de los reportados para Veracruz (Flores-Verdugo et al., 2007) 


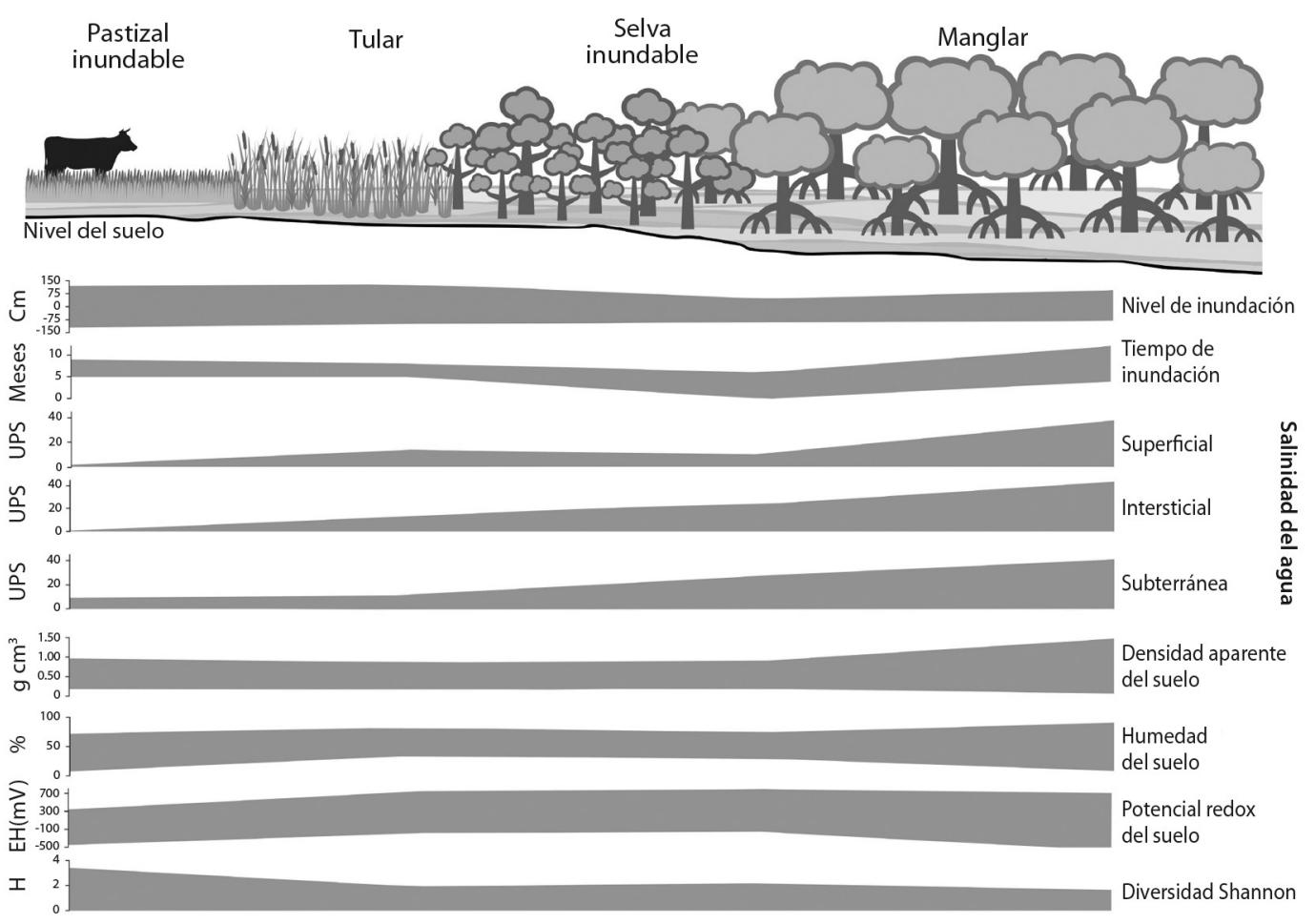

Fig. 5. Distribución de la vegetación a lo largo del gradiente ambiental en el Sistema de Humedales el Castaño.

Fig. 5. Vegetation distribution along the environmental gradient in the El Castaño Wetland System.

y Tabasco (Torres, Infante-Mata, Sánchez, Espinoza-Tenorio, \& Barba, 2017). En el caso del tular y la selva inundable la salinidad es mayor a la observada comúnmente en estos humedales en Veracruz (Yetter, 2004; FloresVerdugo et al., 2007), aunque se han reportado algunos valores más altos, como es el caso del tular por Moreno-Casasola et al. (2010) y las selvas inundables con presencia de $R$. mangle (Infante-Mata et al., 2011; Infante-Mata, Moreno-Casasola, \& Madero-Vega, 2014) en la que la salinidad está dentro del rango registrado en el SHC. Estas diferencias en el Castaño se relacionan con la distancia en que se ubican los tulares y los remanentes de la selva inundable con respecto al mar y durante la temporada de secas es más evidente al presentar los mayores valores de salinidad que llegan por arriba de 15.00 ups. Es por tanto importante considerar la fragilidad de estas comunidades ante los procesos de intrusión salina como efecto del cambio climático (Herbert et al., 2015; Zhai et al., 2018).

El pastizal inundable es el humedal con menor influencia marina, la salinidad es poca, y presenta la mayor diversidad, con la menor diversidad le sigue la selva inundable y el tular y al final el manglar con mayor influencia marina y menor diversidad. Esto significa que al reducirse el estrés por salinidad la diversidad aumenta. El recambio de especies, es mayor entre el manglar y el pastizal inundable, los humedales más alejados y con mayor diferencia de salinidad, y entre el pastizal inundable y la selva inundable con la mayor diferencia de inundación. Por lo que la segunda hipótesis también se cumple parcialmente ya que se esperaba que el tular fuera más diverso que la selva. En el SHC el gradiente de mayor a menor salinidad del manglar hacia los pastizales inundables, es uno de los factores ambientales que están diferenciando la distribución de 
la vegetación. Por lo que los valores más altos de similitud están entre las comunidades vecinas, en este caso el manglar y la selva inundables, y el tular y el pastizal inundable.

Sin embargo, en el pastizal inundable la alta diversidad también está relacionada con las especies estacionales en los cuerpos de agua temporales durante las lluvias y el efecto del disturbio por el uso ganadero (Grime, 1973; Cingolani, Noy-Meir, \& Díaz, 2005). En el pastizal inundable el ganado pasta durante 6 a 8 meses (sequía) evitando que la población de las especies nativas dominantes aumente y permitiendo que las poblaciones de otras especies crezcan (Cingolani, Noy-Meir, Renison, \& Cabido, 2008) y al retirar el ganado durante al menos seis meses por la inundación, permite al suelo recuperarse (MorenoCasasola, López-Rosas, \& Rodríguez-Medina, 2012b; Rodríguez-Medina, Moreno-Casasola, \& Yañez-Arena, 2017), lo que al final aumenta la diversidad. Por lo que mantener el hidroperiodo permite la eliminación y control de ciertas especies, evitando que las poblaciones de especies no nativas se expandan (MorenoCasasola et al., 2018).

En relación con la distribución, el humedal más cercano al mar es el manglar, en el que se encuentran cuatro de las seis especies de mangle registradas para Chiapas, $R$. mangle y L. racemosa son las dos especies dominantes. La densidad (1 945 ind ha-1) y área basal (20.5 $\mathrm{m}^{2} \mathrm{ha}^{-1}$ ) son bajas con respecto a lo observado por Tovilla-Hernández y Romero-Berny (2012) y Romero-Berny et al. (2019) para Chiapas. Considerando solo el área de la Encrucijada Tovilla-Hernández, Aguilar-López, GordilloSolís, Rojas-García y Vazquez-Lule (2010) reportan una densidad similar, aunque un área basal mayor. Comparando con los manglares del Golfo de México la dominancia, la densidad y el área basal es diferente. En Alvarado y la Mancha, Veracruz, dominan Avicennia germinans y $L$. racemosa, con una densidad menor y un área basal mayor (Agraz et al., 2011; Rodriguez-Zuñiga, Ramírez-García, \& Gutiérrez-Granado, 2011) y en la Laguna Mecoacán, Tabasco, domina A. germinans, con una densidad mayor y menor área basal que en el SHC (Torres et al., 2017). Las diferencias de estructura entre los manglares de Chiapas, se relacionan con las variables ambientales como la salinidad, el hidroperiodo, los nutrientes y los impactos antrópicos, en el caso de las diferencias con el Golfo de México también se tienen que considerar la ubicación latitudinal, el clima, y la geomorfología (López-Portillo \& Ezcurra, 2002; López- Portillo et al., 2011; Romero-Berny et al., 2019).

Las especies registradas en el manglar del SHC, coinciden con las reportadas por Tovilla et al. (2007), excepto por el mangle amarillo, Rhizophora harrisonii de distribución limitada en la Encrucijada y Phyllanthus elsiae. También se tienen especies que no están reportadas para el manglar como: $C$. sicyoides, $C$. oaxacana, Hippomane mancinella, aunque se distribuyen en La Encrucijada, (SEMARNAP, 1999). La mayor parte de las especies que acompañan al manglar se establecen en los suelos elevados o en los sitios con influencia de agua dulce.

Siguiendo con la distribución de los humedales, atrás de los manglares están las selvas inundables dominadas por $P$. aquatica, que difieren en estructura con las estudiadas en Veracruz y Tabasco, la densidad es mayor, el área basal es menor, la riqueza y la diversidad son menores (Ascencio, 1994; Infante-Mata et al., 2011; Rincón, 2014). Con respecto a la composición Chiapas y Veracruz comparten 10 especies. Resalta la presencia de Zygia conzattii en Chiapas, con un valor alto de importancia y su ausencia en Veracruz. En ambas selvas hay palmas, en el SHC, S. mexicana y en Veracruz: Attalea liebmannii y Roystonea dunlapiana. Las selvas inundables de México son afines a las de Centro y Sur América, por los géneros y especies en común, (Infante-Mata et al., 2011). Moreno-Casasola, Infante-Mata y López-Rosas (2012a), consideran que también son diversas en composición, debido a la mayor heterogeneidad ambiental de las costas de México (Contreras-Espinoza \& Warner, 2004; LópezPortillo et al., 2011).

En el SHC los tulares son dominados por T. domingensis, ocupando amplias extensiones 
que colindan con los manglares y las selvas inundables en la zona del delta del río San Nicolás y tierra adentro colindan con los humedales modificados como los pastizales inundables, tierras de cultivo temporal, o por asentamientos humanos. Al igual que los tulares de Veracruz, dominados por $T$. domingensis, la composición de especies es baja. Algunas de las especies que comparten son $C$. giganteus, $P$. foetida y $F$. clausum (Peralta-Peláez \& Moreno-Casasola, 2009; Moreno-Casasola et al., 2010).

Los pastizales inundables son los de menor influencia marina en el SHC, son humedales modificados para el uso ganadero. En éstos se observan parches remanentes de vegetación original y colindan principalmente con los tulares. Tienen la mayor riqueza y diversidad de los humedales de estudio y también con respecto a los pastizales inundables de Veracruz estudiados por Travieso-Bello, Moreno-Casasola y Campos (2005) y Rodríguez-Medina et al. (2017). Es una comunidad de herbáceas, dominada por el pasto nativo $P$. conjugatum, especie frecuente en sitios inundados. Hay otros pastos nativos como $P$. virgatum, Hymenachne amplexicaulis, y tres especies de pastos exóticos, siendo $B$. decumbens, el de mayor presencia. En SHC, en los pastizales inundables se mantiene la hidrología y no se desecan, se ha intentado sembrar pasto para forraje, sin embargo las condiciones permiten que siga dominando P. conjugatum.

A parte del pastizal inundable en los otros humedales también se registraron especies no nativas, como plántulas y juveniles de la palma de aceite, E. guineensis, en las selvas inundables y los suelos elevados del manglar. Esta especie es catalogada como invasora por la CONABIO (2017) e Invasive Species Specialist Group ISSG (2015). Se desconocen los efectos que puede tener en los ecosistemas costeros naturales, y su presencia va en aumento (Martínez, Castellanos, \& Guerrero, 2016). En el tular se registró, $B$. decumbens, que consideramos no ha prosperado por su poca tolerancia a la inundación (Holman, Rivas, Argel, \& Pérez, 2004).
Comparando, la costa de Chiapas en el Pacífico con el Golfo de México, este último tiene un mayor espacio de la planicie de inundación, hay una mayor heterogeneidad ambiental, que puede explicar la mayor diversidad de especies en humedales como la selva inundable, en relación con los que existen en la costa de Chiapas (Infante-Mata et al., 2011; López-Portillo et al., 2011; Moreno-Casasola et al., 2012a). La distribución de la vegetación y el funcionamiento de los humedales costeros en parte se explican por el gradiente de los factores ambientales como la salinidad, sin embargo, para entender las diferencias hay que considerar las características hidrogeomorfológicas de cada sitio, que se reflejan en la extensión y el ensamblaje de los ecosistemas costeros (Phillips, 2018). Sin embargo, en México, aún faltan estudios para terminar de comprender totalmente el funcionamiento en los humedales costeros.

En conclusión el gradiente de salinidad de mayor a menor influencia marina es uno de los factores que definen la distribución, composición, diversidad y estructura de la vegetación en el SHC. Se esperaba un gradiente similar de la inundación; sin embargo, esta condición no se cumplió por la dinámica hidrológica que presenta los mayores aportes de agua a los extremos del sistema. La diversidad de cada humedal responde a los cambios en la salinidad y el recambio de especies, diversidad beta, es evidente entre algunos humedales, por lo que existe un cambio de la vegetación que se relaciona con el gradiente ambiental. Finalmente la distribución de la vegetación en el SHC siguen los patrones de organización típica de los humedales costeros tropicales, (Zedler \& Callaway, 2001; Moreno-Casasola et al., 2012a) del litoral hacia tierra adentro se observan los manglares, las selvas inundables y humedales herbáceos, en este caso los tulares y pastizales inundables.

Declaración de ética: los autores declaran que todos están de acuerdo con esta publicación y que han hecho aportes que justifican su autoría; que no hay conflicto de interés de 
ningún tipo; y que han cumplido con todos los requisitos y procedimientos éticos y legales pertinentes. Todas las fuentes de financiamiento se detallan plena y claramente en la sección de agradecimientos. El respectivo documento legal firmado se encuentra en los archivos de la revista.

\section{AGRADECIMIENTOS}

Este trabajo se realizó gracias al apoyo del Consejo Nacional de Ciencia y Tecnología por otorgar la beca no. 239913 al primer autor, la Comisión Nacional de Áreas Naturales Protegidas-Reserva de la Biosfera La Encrucijada, a los habitantes de la comunidad El Castaño, Mapastepec, en particular al grupo Ecoturístico Costa Verde, al grupo de monitores comunitarios de la comunidad de la Palma y a J. Valle Mora por la asesoría en los análisis estadísticos.

\section{RESUMEN}

Introducción: Las características de los humedales costeros son resultado de las interacciones hidrogeomorfológicas entre el continente y el océano, que causan un gradiente ambiental, que resulta en diferentes tipos de vegetación como manglares, popales, tulares, selvas y palmares inundables. Objetivo: Caracterizar las variables del hidroperiodo y fisicoquímicas del agua y suelo para determinar la relación que existe en el patrón de distribución de la vegetación en el Sistema de Humedales El Castaño (SHC). Metodología: Se establecieron 11 unidades de muestreo (UM) permanentes por estrato definidos: cinco en el manglar, dos en selvas inundables, dos en tular y dos en pastizal inundable. De mayo 2016 a octubre 2017 se caracterizó la vegetación y se muestreó mensualmente los niveles de inundación y parámetros fisicoquímicos del agua (superficial, intersticial y subterránea): salinidad, conductividad y $\mathrm{pH}$; y el suelo: densidad aparente, porcentaje de humedad y potencial redox. Resultados: El manglar es el más cercano al mar, tiene la menor diversidad (H:1.66) y especies registradas (14), está dominado por Laguncularia racemosa y Rhizophora mangle y tiene los valores más altos de salinidad intersticial y subterránea, mayores a 10.8 ups, se mantiene inundado de 4 a 12 meses, su potencial redox es de $14.57 \mathrm{mV}$. Seguido está el manglar, tierra adentro, se ubican los remanentes de la selva inundable, (H:2.18 y 18 especies), dominada por Pachira aquatica, la salinidad intersticial y subterránea de 4.95 ups, permanece inundada de 0 a 6 meses y el potencial redox es de 119.07 $\mathrm{mV}$. El tular, después de la selva, (H:1.92 y 16 especies), dominado por Typha domingensis, salinidad intersticial y subterránea de 6.1 ups, el tiempo de inundación es de 5 a 8 meses y potencial redox es de $125.9 \mathrm{mV}$. El pastizal inundable, con menor influencia marina, es un humedal herbáceo modificado para uso ganadero, presentó los valores más altos de diversidad (H:3.44 y 50 especies), Paspalum conjugatum es la especie dominante, la salinidad intersticial y subterránea es menor a 0.5 ups, se mantiene inundado de 5 a 9 meses y el potencial redox es de 151.23 $\mathrm{mV}$. Conclusiones: En cada tipo de vegetación, la estructura, composición y diversidad es diferente, con un alto recambio de especies que indica un gradiente definido por la salinidad. La vegetación en el SHC sigue los patrones de organización típica de los humedales costeros tropicales, manglares, selvas inundables y humedales herbáceos, en este caso los tulares y pastizales inundables. El factor que define la distribución de la vegetación, es salinidad y el gradiente que se observa está en función de la dinámica hidrológica que depende de entradas de agua marina y de la bajada de agua dulce del interior del continente.

Palabras clave: gradiente ambiental, hidrófilas, turbera tropical, humedal modificado, hidrología, diversidad, conectividad.

\section{REFERENCIAS}

Agraz-Hernández, C.M., García-Zaragoza, C., IriarteVivar, S., Flores-Verdugo, F.J., \& Moreno-Casasola, P. (2011). Forest structure, productivity and species phenology of mangroves in the La Mancha lagoon in the Atlantic coast of Mexico. Wetlands Ecology and Management, 19, 273-293.

Alonso-EguíaLis, P., Brena, J., Castillo, C., Martínez, M., Sánchez, E., Vara, E., ... Vera, J. (2013). Inventario y programa de manejo integral para la preservación de los humedales del Soconusco a través de su delimitación, caracterización ecológica, hidrológica, social y grado de riesgo (Reporte técnico). México, DF: Fondo de Investigación sobre Agua, Conacyt-Conagua.

Anderson, C.J., \& Lockaby, G. (2011). Forested Wetland Communities as Indicators of Tidal Influence along the Apalachicola River, Florida, USA. Wetlands, 31, 895-906. DOI: 10.1007/s13157-011-0204-5

Aponte, H. (2017). Diversidad beta en los humedales costeros de lima, perú: estimación con Índices de presencia/ausencia y sus implicancias en conservación. The Biologist (Lima), 15(1), 9-14.

Ascencio, R. (1994). Estructura y composición florística de una selva baja inundable de Pachira aquatica Aubl. (Apompal) en Ogarrio, Huimanguillo, Tabasco, México (Tesis de licenciatura). Universidad Juárez Autónoma de Tabasco, Tabasco. 
Barendregt, A., \& Swarth, C.W. (2013). Tidal Freshwater Wetlands: Variation and Changes. Estuaries and Coasts, 3, 445-456.

Bergh, V.D., Bartender, J.A., Gilbert, A., van Herwijnen, M., van Horssen, P., Kandelaars, P., \& Lorenz, C. (2001). Spatial economic-hydroecological modelling and evaluation of land use impacts in the Vecht wetlands area. Environmental Modeling and Assessment, 6, 87-100.

Breedlove, D.E. (1981). Flora of Chiapas, part I: Introduction to the Flora of Chiapas. San Francisco: The California Academy of Sciences.

Brinson, M., Bradshaw, H.D., \& Jones, M.N. (1985). Transitions in forested wetlands along gradients of salinity and hydroperiod. Journal of the Elisha Mitchell Scientific Society, 101(2), 76-94.

Calva, L., Pérez, A., \& Márquez, A. (2006). Contenido de carbono orgánico y características texturales de los sedimentos del sistema costero lagunar ChantutoPanzacola. Hidrobiológica, 16(02), 127-136.

Carranza, J., \& Molina, C. (2003). Estimación de la tasa de transformación del hábitat en la Reserva de la Biosfera la Encrucijada, Periodo 1975-2000 (Informe técnico). México: Comisión Nacional de Áreas Naturales Protegidas y el Fondo Mexicano para la Conservación de la Naturaleza A.C.

Carreño, M.F., Esteve, M.A., Martínez, J., Palazón, J.A., \& Pardo, M.T. (2008). Habitat change in coastal wetlands associated to hydrological changes in the watershed. Estuarine, Coastal and Shelf Science, 77, 475-483.

Cingolani, A.M., Noy-Meir, I., \& Díaz, S. (2005). Grazing effects on rangeland diversity: a synthesis of contemporary models. Ecological Applications, 15, 757-773.

Cingolani, A.M., Noy-Meir, I., Renison, D., \& Cabido, M. (2008). La ganadería extensiva, ¿es compatible con la conservación de la biodiversidad y de los suelos? Ecología Austral, 18, 253-271.

CONABIO (Comisión Nacional para el Conocimiento y Uso de la Biodiversidad). (2016). EncicloVida. México: CONABIO. Recuperado de www.enciclovida.mx

CONABIO (Comisión Nacional para el Conocimiento y Uso de la Biodiversidad). (2017). Evaluación rápida de invasividad de Elaeis guineensis Jacq., 1763. Sistema de información sobre especies invasoras en México (Informe). México: Comisión Nacional para el Conocimiento y Uso de la Biodiversidad.

CONAGUA-SMN (Comisión Nacional del Agua-Sistema Meteorológica Nacional). (2018). Estación meteorológica 7344. Comisión Nacional del Agua, Sistema Meteológico Nacional. Recuperado de http://smn.cna. gob.mx/es/estaciones-meteorologicas-automaticas-2
Contreras-Espinosa, F., \& Warner, G. (2004). Ecosystem characteristics and management considerations for coastal wetlands in Mexico. Hydrobiologia, 511, 233-245.

Costanza, R., d'Arge, R., de Groot, R.S., Farber, S., Grasso, M., Hannon, B., ... van den Belt, M. (1997). The value of the world's ecosystem services and natural capital. Nature, 387, 253-260.

Courtwright, J., \& Findlay, S.E.G. (2011). Effects of Microtopography on Hydrology, Physicochemistry, and Vegetation in a Tidal Swamp of the Hudson River. Wetlands, 31, 239. DOI: 10.1007/s13157-011-0156-9

Crain, C.M., Silliman, B.R., Bertness, S.L., \& Bertness, M.D. (2004). Physical and biotic drivers of plant distribution across estuarine salinity gradients. Ecology, 85(9), 2539-2549.

Crase, B, Liedloff, A., Vesk, P.A., Burgman, M., \& Wintle, B.A. (2013). Hydroperiod is the main driver of the spatial pattern of dominance in mangrove communities. Global Ecology and Biogeograph, 22, 806-817.

Davis, S., Childers, D., Lorenz, J., Wanless, H., \& Hopkins, T., (2005). A conceptual model of ecological interactions in the mangrove estuaries of the Florida Everglades. Wetlands, 25(4), 832-842.

de Groot, R., Brander, L., van der Ploeg, S., Costanza, R., Bernard, F., Braat, L., ... van Beukering, P. (2012). Global estimates of the value of ecosystems and their services in monetary units. Ecosystem Services, 1, 50-61.

de la Fuente, J., Lisle, T. E., \& McKean, J., (2007). Landslides, Channel Erosion, and Sedimentation in the Western Sierra Madre, Chiapas, Mexico, During Hurricane Stan in 2005: A Brief Field Review with Recommendations (Technical report). USA: USAID and US Forest Service.

de La Lanza Espino, G., Pérez, M.A.O., \& Pérez, J.L.C. (2013). Diferenciación hidrogeomorfológica de los ambientes costeros del Pacífico, del Golfo de México y del Mar Caribe. Investigaciones Geográficas, 81, 33-50.

Elmore, J.A., Mustard, J., \& Manning, S. (2003). Regional patterns of plant community response to changes in water: owens Valley, California. Ecological Applications, 13(2), 443-460.

Flores-Verdugo, F., Moreno- Casasola, P., Agraz, M.C., López, H., Benítez, D., \& Travieso, C. (2007). La topografía y el hidroperiodo: dos factores que condicionan la restauración de los humedales costeros. Boletín de la Sociedad Botánica Mexicana, 80(Suppl.), 33-47.

Fourqurean, J., Johnson, B., Kauffman, J.B., Kennedy, H., Lovelockand, C., Emmer, I., ... Serrano, O. (2014). Field sampling of soil carbon pool in the coastal 
ecosystems. In J. Howard, S. Hoyt, K. Isensee, E. Pidgeon, \& M. Telszewski (Eds.), Coastal Blue Carbon: Methods for assessing carbon stocks and emissions factors in mangroves, tidal salt marshes, and seagrass meadows (pp. 39-66). Conservation International, Intergovernmental Oceanographic Commission of UNESCO, International Union for Conservation of Nature, Arlington, Virginia, USA.

García, E. (2004). Modificaciones al Sistema de Clasificación Climática de Köppen (Serie de Libros No. 6). México: Instituto de Geografía de la UNAM.

Givnish, T.J., Volin, J.C., Owen, V.D., Volin, V.C., Muss, J.D., \& Glaser, P.H. (2008). Vegetation differentiation in the patterned landscape of the central Everglades: importance of local and landscape drivers. Global Ecology and Biogeograph, 17, 384-402.

Grime, J.P. (1973). Competitive exclusion in herbaceous vegetation. Nature, 242, 344-347.

Hackney, C.T., \& Avery, G.B. (2015). Tidal Wetland Community Response to Varying Levels of Flooding by Saline Water. Wetlands, 35, 227. DOI: 10.1007/ s13157-014-0597-z

Hammer, Ø., Harper, D.A.T., \& Ryan, P.D. (2001). Paleontological Statistics software package for education and data analysis. Recuperado de https://palaeoelectronica.org/2001_1/past/past.pdf

Herbert, E.R., Boon, P., Burgin, A.J., Neubauer, S.C., Franklin, R.B., Ardón, M., ... Gell, P. (2015). A Global perspective on wetlands salinization: ecological consequences of a growing threat to freshwater wetlands. Ecosphere, 6, 1-43.

Holmann, F., Rivas, L., Argel, P., \& Pérez, E. (2004). Impacto de la adopción de pastos Brachiaria: Centroamérica y México (Documento de Trabajo No. 197). Centro Internacional de Agricultura Tropical (CIAT). Recuperado de http://lrrd.cipav.org.co/ lrrd16/12/holm16098.htm

Infante-Mata, D., Moreno-Casasola, P., \& Madero-Vega, C. (2014). ¿Pachira aquatica, un indicador del límite del manglar? Revista Mexicana de Biodiversidad, $85,143-160$.

Infante-Mata, D., Moreno-Casasola, P., Madero-Vega, C., Castillo-Campos, G., \& Warner, G. (2011). Floristic composition and soil characteristics of tropical freshwater forested wetland of Veracruz on the coastal plain of the Gulf of Mexico. Forest Ecology and Management, 262, 1514-531.

Infante-Mata, D, Peralta-Pélaez, L.A., \& Arrocha, A. (2009). Obtención de datos de salinidad, conductividad y $\mathrm{pH}$ del agua. In P. Moreno-Casasola \& B. Warner (Eds.), Breviario para describir, observar y manejar humedales (pp. 31-42). Veracruz, México: Serie Costa Sustentable No. 1. RAMSAR Instituto de Ecología A.C., CONANP, US Fish.
Invasive Species Specialist Group ISSG (2015). Global Invasive Species Database Especie: Elaeis guineensis (Versión 2015.1). Recuperado de http://www. iucngisd.org/gisd/search.php

Kauffman, J.B., Donato, D.C., \& Adame, M.F. (2013). Protocolo para la medición, monitoreo y reporte de la estructura, biomasa y reservas de carbono de los manglares (Documento de Trabajo 117). Bogor, Indonesia: CIFOR.

King, R.S., Richardson, C., Urban, D.L., \& Romanowicz, E.A. (2004). Spatial Dependency of Vegetation-Environment Linkages in an Anthropogenically Influenced Wetland. Ecosystem Ecosystems, 7, 75-97.

Landgrave, R., \& Moreno-Casasola, P. (2012). Evaluación cuantitativa de la pérdida de humedales en México. Investigación Ambiental, 4(1),19-35.

Lee, S.Y., Jones, E.B.G., Diele, K., Castellanos-Galindo, G.A., \& Nordhaus, I. (2017). Biodiversity. In V.H. Rivera-Monroy, S.Y. Lee, E Kristensen, \& R.R. Twilley (Eds.), Mangrove Ecosystems: A Global Biogeographic Perspective Structure, Function, and Services (pp. 55-86). Cham, Switzerland. Springer. DOI: $10.1007 / 978-3-319-62206-4$

Liscovsky, I.J., Parra, M.V., Balente, O.H., Huerta, M., \& Ramos, P. (2009). Diagnóstico comunitario participativo El Castaño, Mapastepec, Chiapas (Reporte). México: El Colegio de la Frontera Sur-San Cristóbal de las Casas.

Lisle, T.E., De la Fuente, J., Suarez, C., \& Hernández, A. (2009). An Overview of Watershed Management Issues in Coastal Chiapas: Report of a Technical Visit (Technical report). USA: USAID and US Forest Service.

López-Portillo, J., \& Ezcurra, E. (2002). Los manglares de México: una revisión. Madera y Bosques, No. Esp. 27-51.

López-Portillo, J., Martínez, M.L., Hesp, P., Hernández, J.R., Méndez, A.P., Vasquez-Reyes, V., ... Ganchuz, D.S.L. (2011). Atlas de las costas de Veracruz, manglares y dunas. Veracruz, México: Secretaria de Educación del Estado de Veracruz, Universidad Veracruzana.

López-Rosas, R.H., \& Tolome, J. (2009). Medición del potencial redox del suelo y construcción de electrodos de platino. In P. Moreno-Casasola \& B. Warner (Eds.), Breviario para describir, observar y manejar humedales (pp. 131-138). Veracruz, México: Serie Costa Sustentable No. 1. RAMSAR Instituto de Ecología A.C., CONANP, US Fish.

Martínez, M.P., Castellanos-Navarrete, A., \& Guerrero, S. (2016). Fortalecimiento a la estrategia de control de palma africana en la Reserva de la Biosfera La Encrucijada Convenio PROCER/CCER/RFSIPS/31/2016 (Informe interno). México: Naturaleza y Redes AC. 
Comisión Nacional de Áreas Naturales ProtegidasReserva de la Biosfera La Encrucijada.

MEA (Millennium Ecosystem Assessment). (2005) Ecosystems and human well-being. Island Press: Washington D.C.

Mitsch, W.J., \& Gosselink, J.G. (2007). Wetlands. New York, USA: John Wiley and Sons.

Moreno-Casasola, P., Cejudo-Espinosa, E., CapistránBarradas, A., Infante-Mata, D., López-Rosas, H., ... Campos-Cascaredo, A. (2010). Composición florística, diversidad y ecología de humedales herbáceos emergentes en la planicie costera central de Veracruz, México. Boletín de la Sociedad Botánica de México, 87, 29-50.

Moreno-Casasola, P., Hernández, M.E., Campos, A., Vázquez-González, C., López-Rosas, H., Peralta-Peláez, L.A., \& Monroy, R. (2018). La ganadería en los humedales: ¿tiene futuro? En G. Halffter, M. Cruz, \& C. Huerta (Comps.), Ganadería sustentable en el Golfo de México (pp. 285-314). Instituto de Ecología, A. C., México.

Moreno-Casasola, P., Infante-Mata, D., \& López-Rosas, H. (2012a). Tropical freshwater marshes and swamps of North America. In D. Batzer (Ed.), Wetland habitats of North America: Ecology and Conservation Concerns (pp. 267-282). Berkeley: University of California Press.

Moreno-Casasola, P., López-Rosas, H., \& RodríguezMedina, K. (2012b). From tropical wetlands to pastures on the coast of the Golf of Mexico. Pastos, 42(2), 185-217.

Moreno-Casasola, P., \& Warner, B. (Eds.). (2009). Breviario para describir, observar y manejar humedales. Serie Costa Sustentable no 1. Veracruz, México: RAMSAR, Instituto de Ecología A.C., CONANP, US Fish and Wildlife Service, US State Department.

Peralta-Pélaez, L.A., Infante-Mata, D., \& Moreno-Casasola, P. (2009). Construcción e instalación de piezómetro. In P. Moreno-Casasola \& B. Warner (Eds.), Breviario para describir, observar y manejar humedales. Serie Costa Sustentable (No. 1, pp.17-39). Veracruz, México: RAMSAR Instituto de Ecología A. C., CONANP, US Fish and Wildlife Service, US State Department.

Peralta-Pélaez, L.A., \& Moreno-Casasola, P. (2009). Composición florística y diversidad de la vegetación de Humedales en los lagos interdunarios de Veracruz. Boletín de la Sociedad Botánica de México, 85, 89-101.

Pérez, N.S. (2013). Erosión hídrica en cuencas costeras de Chiapas y estrategias para la restauración hidrológico-ambiental (Tesis de doctorado). Colegio de Posgraduados - Campus Montecillo, Texcoco Estado de México.
Phillips, J.D. (2018). Environmental gradient and complexity in coastal landscape response to sea level rise. Catena, 169, 107-118.

R Core Team. (2019). R: A Language and environment for statistical computing. Vienna, Austria: R Foundation for Statistical Computing. Recuperado de https:// www.r-project.org

Rickert, C., Fichtner, A., van Klink, R., \& Bakker, J.P. (2012). $\alpha$ - and $\beta$-diversity in moth communities in salt marshes is driven by grazing management. Biological Conservation, 146, 24-31.

Rincón, P.M. (2014). Bosques de zapotonales (Pachira aquatica) en la Reserva de la Biosfera La Encrucijada, Chiapas, México (Tesis maestría). Instituto de Ecología de la UNAM, México D. F.

Rodríguez-Medina, K., Moreno-Casasola, P., \& YañezArenas, C. (2017). Efecto de la ganadería y la variación estacional sobre la composición florística y la biomasa vegetal en los humedales de la costa centro oeste del Golfo de México. Acta Botánica Mexicana, 119, 79-99.

Rodríguez-Zuñiga, M.T., Ramírez-García, P., \& GutiérrezGranados, G. (2011). Efectos de la extracción no controlada de madera sobre la comunidad y estructura de tamaños de los manglares de Alvarado, Veracruz, México. Boletín de la Sociedad Botánica de México, 89, 107-113.

Romero-Berny, E., Tovilla-Hernández, C., TorrescanoValle, N., \& Schmook, B. (2019). Heterogeneidad estructural del manglar como respuesta a factores ambientales y antrópicos en el Soconusco, Chiapas, México. Polibotánica, 47, 1-20.

Rzedowski, J. (1978). Vegetación de México. México: Edit. Limusa.

SEMARNAP (Secretaria de Medio Ambiente Recursos Naturales y Pesca). (1999). Programa de Manejo de la Reserva de la Biosfera La Encrucijada. México D. F: Instituto Nacional de Ecología.

Socolar, J.B., Gilroy, J.J., Kunin, W.E., \& Edwards, D.P. (2016). How Should Beta-Diversity Inform Biodiversity Conservation? Trends in Ecology and Evolution, 31(1), 67-80.

Torres, J.R., Infante-Mata, D., Sánchez, A.J., EspinozaTenorio, T., \& Barba, E. (2017). Atributos estructurales, productividad (hojarasca) y fenología del manglar en la Laguna Mecoacán, Golfo de México. Revista Biología Trópical, 65(4), 1592-1608.

Tovilla, H.C., Salas-Roblero, R.L., De La Presa-Pérez, J.C., Romero-Berny, E., Ovalle-Estrada, F., GómezOrtega A., ... Hernández-Guzmán, A. (2007). Inventario forestal de los bosques de manglar de la costa de Chiapas (Informe final). Chiapas, México: ECOSUR-COCYTECH. 
Tovilla-Hernández, C., Aguilar-López, E., Gordillo-Solís, O.G., Rojas-García, J., \& Vázquez-Lule, J. (2010). Caracterización del sitio de manglar La Encrucijada. En Sitios de manglar con relevancia biológica y con necesidades de rehabilitación ecológica. México: CONABIO.

Tovilla-Hernández, C., \& Romero-Berny, E.I. (2012). Diagnóstico estructural de los manglares de Chiapas y Oaxaca. In A. J. Sánchez, X. Chiappa-Carrara, \& R. Brito-Pérez (Eds.), Recursos acuáticos costeros del sureste (Vol. 1, pp. 257-279). Mérida: FOMIXCONCITEY, RECORECOS, UNAM.

Travieso-Bello, A., Moreno-Casasola, P., \& Campos, A. (2005). Efecto de diferentes manejos pecuarios sobre el suelo y la vegetación en humedales transformados a pastizales. Interciencia, 30(1), 12-18.

UICN (International Union for Conservation of Natural). (2017). Directrices de uso de las Categorías y Criterios de la Lista Roja UICN (Versión 13). Recuperado de http://www.iucnredlist.org/documents/RedListGuidelines.pdf

Valderrama-Landeros L. H., Rodríguez-Zúñiga, M.T., Troche-Souza C., Velázquez-Salazar, S., Villeda-Chávez, E., Alcántara-Maya, J.A., ... Ressl, R. (2017). Manglares de México: actualización y exploración de los datos del sistema de monitoreo 1970/1980-2015. Ciudad de México: Comisión Nacional para el Conocimiento y Uso de la Biodiversidad.

Vepraskas, M.J., \& Faulkner, S.P. (2001). Redox chemistry of hydric soils, In J.L. Richardson \& M.J. Vepraskas (Eds.), Wetland Soils. Genesis, hydrology, landscapes, and classification. Boca Raton: Lewis Publishers, CRC Press.
Westhoff, V., \& Van der Maarel, E. (1978). The BraunBlanquet Approach. In R. Whittaker (Ed.), Classification of Plant Communities. The Hague: Kluwer Academic Publishers.

Wheeler, B.D., Money, R.P., Shaw, S.C., Perrow, M.R., \& Davy A.J. (2002). Freshwater Wetlands. In M.R. Perrow \& A.J. Davy (Eds.), Handbook of Ecological Restoration (Vol. 2) (pp. 325-354). Cambridge, Unitet Kindong: Cambridge UniversityPress.

Wilke, B.M. (2005). Determination of Chemical and Physical Soil Properties. In R. Margesin \& F. Schinner (Eds.), Manual for Soil Analysis: Monitoring and Assessing Soil Bioremediation (pp. 47-96). Berlin: Springer.

Yetter, J.C. (2004). Hydrology and geochemistry of freshwater wetlands on the gulf coast of Veracruz, Mexico (Tesis de maestría). University of Waterloo, Ontario, Canada.

Zhai, L., Krauss, K.W., Liu, X., Duberstein, J.A., Duberstein, J.A., Conner, W.H., ... Sternberg, L.d.S.L. (2018). Growth stress response to sea level rise in species with constrasting functional traits: A case study in tidal freshwater forested wetlands. Environmental and Experimental Botany, 155, 378-386.

Zedler, J.B., \& Callaway, J.C. (2001). Tidal Wetland Restoration: Physical and Ecological processes. Journal of Coastal Research, 27, 38-64.

Zeilhofer, P., \& Schessl, M. (1999). Relationship between vegetation and environmental conditions in the northern Pantanal of Mato Grosso, Brazil. Journal of Biogeography, 27, 159-168. 